\title{
Correction to: Reconstructing the Historic Landscape of Larochette, Luxembourg
}

Marleen de Kramer(D), Sam Mersch (iD), and Christopher Morse (iD

\section{Correction to: \\ Chapter "Reconstructing the Historic Landscape of Larochette, Luxembourg" in: M. Ioannides et al. (Eds.): Digital Heritage, LNCS 11197, https://doi.org/10.1007/978-3-030-01765-1_4}

The original version of chapter 4 starting on p. 30 was revised. Inadvertently the funding institution was not mentioned in the original chapter. A footnote for the explanation was added on the first page of the chapter. The original chapter was corrected. 